International Journal of Modern Physics D, Vol. 0, No. 0 (1993) 000-000

(C) World Scientific Publishing Company

\title{
LOCAL MODES, LOCAL VACUUM, LOCAL BOGOLJUBOV COEFFICIENTS AND THE RENORMALISED STRESS TENSOR
}

\author{
S.MASSAR* \\ Service de Physique Théorique, Université Libre de Bruxelles \\ Campus Plaine, C.P. 225, Bd du Triomphe, B-1050 Brussels, Belgium
}

Received (received date)

Revised (revised date)

\begin{abstract}
Local modes and local particles are defined at any point in curved space time as those that most resemble Minkowsky modes at that point. It is shown that the renormalised stress tensor is the difference of energy between the physical vacuum and that defined by these local modes.
\end{abstract}

\section{Introduction}

Some difficulties have arisen in giving a precise meaning to the notion of particles in curved space time幽 (for a review on the subject of quantum fields in curved space-time see 4 ). These are due to the curvature of space, which creates particles and thus generates an uncertainty in their number.

In this article (which generalises some previous ideas of ours ) we try to clarify this issue. We proceed via the construction at an arbitrary point in space time $\mathcal{P}$ of a set of local modes chosen so as to most closely resemble the Minkowskian ones. These modes then describe the local particles. The justification of our procedure stems from the fact that the value of the renormalised energy momentum tensor at $\mathcal{P}$ is the energy momentum of the local particles at $\mathcal{P}$ (up to an anomalous term depending only on the geometry at $\mathcal{P}$ ).

Since the local modes are those which an inertial observer at $\mathcal{P}$ would most naturally choose as a basis for quantisation, it appears that the gravitational field strongly resembles inertial particle detectors, being insensitive to the infinite energy density in the local vacuum and responding only to the excitations thereof.

The number of particles present at the space time point $\mathcal{P}$ under consideration is given a precise meaning by introducing the (local) Bogoljubov coefficients between the local modes and the physical modes. In this way, contact is made with the usual manner of exhibiting particle creation through the Bogoljubov coefficients between asymptotic modes (as in Hawking's original derivation of black hole radiance $\sqrt{ }$ ), since in flat regions of space time the local modes and the asymptotic modes coincide.

*Boursier I.I.S.N., e-mail: smassar@ulb.ac.be 
Local Modes, Local Vacuum, Local Bogoljubov Transformations and...

\section{Two Different Vacuum States}

As a preparatory exercise we first recall how two different quantisations of a scalar field are related by Bogoljubov coefficients. Let $\phi$ be a real scalar field obeying the equation

$$
\square \phi+m^{2} \phi+\xi R \phi=0
$$

and let $f_{k}(x)$ and $g_{l}(x)$ be two complete orthonormalised sets of solutions of (11). The operator $\phi$ may then be decomposed either as

$$
\phi=\sum_{k} a_{k} f_{k}+a_{k}^{+} f_{k}^{*}
$$

or as

$$
\phi=\sum_{l} b_{l} g_{l}+b_{l}^{+} g_{l}^{*}
$$

The corresponding vacuum states $\mid O_{f}>$ and $\mid O_{g}>$ are annihilated by the $a_{k}$ and $b_{l}$ respectively. The Bogoljubov coefficients relating these two bases are given by

$$
\alpha_{k l}=<f_{k}, g_{l}>\quad \beta_{k l}=<f_{k}^{*}, g_{l}>
$$

where $\langle,>$ is the Klein-Gordon inner product. The $\alpha$ and $\beta$ coefficients obey unitary relations

$$
\alpha^{2}-\beta^{2}=1 \quad \alpha \beta^{*}-\alpha^{*} \beta=0
$$

The propagators $G_{f}\left(x, x^{\prime}\right)=<O_{f}\left|\phi(x) \phi\left(x^{\prime}\right)\right| O_{f}>$ and $G_{g}\left(x, x^{\prime}\right)=<O_{g}\left|\phi(x) \phi\left(x^{\prime}\right)\right| O_{g}>$ are singular at the coincidence point but their difference $G_{f}-G_{g}$ is not. This is a consequence of Hadamard's theorem, 6 which states that Green's functions of Eq. (11) always have the same singular part. Similarly, the difference of energy between the $f$ and $g$ vacuum is finite and is given by

$$
<O_{f}\left|T_{\mu \nu}(x)\right| O_{f}>-<O_{g}\left|T_{\mu \nu}(x)\right| O_{g}>=\lim _{x^{\prime} \rightarrow x} \hat{T}_{\mu \nu}\left(G_{f}\left(x, x^{\prime}\right)-G_{g}\left(x, x^{\prime}\right)\right)
$$

where $\hat{T}_{\mu \nu}$ is a differential operator whose explicit form we do not need here. We note for future use that the difference of energy (6) is a conserved quantity. This is classicaly true and results here from the fact that $G_{f}-G_{g}$ obeys (11) in both variables and is non singular.

The difference of the propagators can be expressed in terms of the $g$ modes and the Bogoljubov coefficients (i.e. in terms of the number of $g$ particles present in the $f$ vacuum):

$$
G_{f}\left(x, x^{\prime}\right)-G_{g}\left(x, x^{\prime}\right)=\int d l d l^{\prime} d k 2 \mathcal{R} e\left[\beta_{l k} \beta_{l^{\prime} k}^{*} g_{l}^{*}(x) g_{l^{\prime}}\left(x^{\prime}\right)-\alpha_{l k} \beta_{l^{\prime} k}^{*} g_{l}(x) g_{l^{\prime}}\left(x^{\prime}\right)\right]
$$

where we have used the unitarity relations (6). Acting on (17) with $\hat{T}_{\mu \nu}$ and taking the coincidence point limit yields an expression for the difference of energies as a function of the Bogoljubov coefficients. 


\section{Local Modes, Local Vacuum, Local Bogoljubov Coefficients and the Renormalised Stress Tensor}

We now turn to the problem at hand, namely the definition of the local quanta and the local vacuum. To this end choose a specific point $\mathcal{P}$ in space time and build Riemann normal coordinates $y^{\mu}$ centred on $\mathcal{P}$ (i.e. the coordinate lines are the geodesics passing through $\mathcal{P}$ ). In these coordinates the metric is given by

$$
g_{\mu \nu}=\eta_{\mu \nu}+\frac{1}{3} R_{\mu \alpha \nu \beta} y^{\alpha} y^{\beta}+O\left(R^{2}\right)
$$

and the wave equation (11) takes the form

$$
\left(\eta_{\mu \nu} \partial_{\mu} \partial_{\nu}+m^{2}+\xi R++\frac{1}{3} R_{\alpha}^{\nu} y^{\alpha} \partial_{\nu}-\frac{1}{3} R_{\alpha \beta}^{\mu \nu} y^{\alpha} y^{\beta} \partial_{\mu} \partial_{\nu}+O\left(R^{2}\right)\right) \phi=0
$$

The local modes are the complete set of solutions of (9) that start off as Minkowsky modes. They can be computed order by order in the curvature:

$$
\begin{array}{r}
\phi_{\text {local } k}=\frac{e^{i k_{\mu} y^{\mu}}}{\sqrt{(2 \pi)^{3} k_{0}}}\left(1+\frac{\xi R}{m^{2}}-\frac{i}{3 m^{2}} R_{\alpha}^{\nu} y^{\alpha} k_{\nu}-\frac{i}{3 m^{2}} R_{\alpha \beta}^{\mu \nu} y^{\alpha} y^{\beta} k_{\mu} k_{\nu}+\right. \\
\left.\quad+\frac{4 i}{3 m^{4}} R_{\alpha}^{\mu \nu \sigma} y^{\alpha} k_{\mu} k_{\nu} k_{\sigma}+\frac{8}{3 m^{8}} R^{\mu \nu \sigma \rho} k_{\mu} k_{\nu} k_{\sigma} k_{\rho}+O\left(R^{2}\right)\right)
\end{array}
$$

Having defined the local modes we proceed exactly as in the preceding section: the states of interest are the local vacuum $\mid O_{\text {local }}(\mathcal{P})>$ and the physical state (taken for simplicity to be a vacuum state $\mid O_{i n}>$ ).

The propagator in the local vacuum is

$$
G_{\text {local }}\left(y^{\mu}, y^{\prime \mu}\right)=<O_{\text {local }}(\mathcal{P})\left|\phi\left(y^{\mu}\right) \phi\left(y^{\prime \mu}\right)\right| O_{\text {local }}(\mathcal{P})>=\int d^{3} k \phi_{\text {local } k}\left(y^{\mu}\right) \phi_{\text {local } k}^{*}\left(y^{\prime \mu}\right)
$$

Note that the local vacuum and the local propagator are parametrised by the space time point $\mathcal{P}$ and will in general change as $\mathcal{P}$ changes.

The renormalised stress tensor is the difference of energy between the physical state and the local vacuum

$$
\begin{aligned}
<T_{\mu \nu}(\mathcal{P})>_{r e n}= & \lim _{\substack{y^{\mu} \rightarrow 0 \\
y^{\prime \mu} \rightarrow 0}} \hat{T}_{\mu \nu}\left[<O_{\text {in }}\left|\phi\left(y^{\mu}\right) \phi\left(y^{\prime \mu}\right)\right| O_{\text {in }}>-\right. \\
& \left.-<O_{\text {local }}(\mathcal{P})\left|\phi\left(y^{\mu}\right) \phi\left(y^{\prime \mu}\right)\right| O_{\text {local }}(\mathcal{P})>\right]
\end{aligned}
$$

Eq. (12) coincides with the usual expressions for $\left\langle T_{\mu \nu}\right\rangle_{r e n}$ found in the literature. This can be readily seen by considering the function $G_{l o c a l}\left(y^{\mu}, 0\right)$ given by (11) with $y^{\prime} \overline{\overline{1}} 0$, which coincides with the adiabatic propagator defined by Bunch and Parkerl (both functions are solutions of the same equation and are built as a series in the curvature starting with the Minkowskian form, therefore they are identical). Bunch and Parker's $\$$ proof of the equivalence of their renormalisation 
procedure and the renormalisation of the DeWitt-Schwinger effective action then carries through unaltered.

As written above, the renormalised stress tensor (12) is not conserved. This arises on taking the divergence of the right hand side of (12) from the space-time dependence of the local vacuum $\mid O_{\text {local }}(\mathcal{P})>$. This situation must be compared with the difference in energy of two fixed states (6), which is conserved. A conserved renormalised stress tensor may be recovered by adding to (12) a term depending only on $m^{2}, \xi$ and the geometry at $\mathcal{P}$ (since the subtraction in (12) depends only on these quantities). 10 In the conformally coupled case this term, the anomaly, depends only on the geometry at $\mathcal{P}$ and restores energy conservation at the expense of conformal invariance, i.e. the stress tensor now has a trace.

Finally, Bogoljubov coefficients between the modes describing the physical state and the local modes can be constructed and the renormalised stress tensor expressed in terms of these coefficients, that is, in terms of the number of local particles present in the physical state.

\section{One Dimensional Case and Black Hole Evaporation}

The above formalism has been applied to the situation of a massless field in $1+1$ dimensions. In that case the series for the local modes (11) may be summed in closed form. After some manipulations the renormalised stress tensor can be expressed as a sum over poles. This result has in turn been used in the effective one-dimensional problem describing black hole evaporation. It then appears that in the part of the stress tensor describing the outgoing flux the poles tend to their thermal value at infinity but differ from it for finite distances, thereby illustrating how the Hawking radiation builds up to its asymptotic value.

\section{Acknowledgements}

The author wishes to thank R. Brout, R. Parentani and Ph. Spindel for important discussions and R. Parentani for a careful reading of the manuscript.

\section{References}

1. S.W. Hawking, Commun. math. Phys. 43, 199 (1975).

2. P. Hájíček, Nuovo Cimento 33 B, 597 (1976).

3. P. Hájíček, Phys. Rev. D 15, 2757 (1977).

4. N.D. Birrel and P.C.W. Davies, Quantum Fields in Curved Space, Cambridge University Press , Cambridge, England (1982).

5. S. Massar, R. Parentani, R. Brout, Brussels preprint ULB-TH-01/93, hep-th 9303147

6. J. Hadamard, Lectures on Cauchy's Problem in Linear Partial Differential Equations, Yale University Press, New Haven, 1923

7. M.R. Brown and A.C. Ottewill, Phys. Rev. D 34, 1776 (1986)

8. T.S. Bunch and L. Parker, Phys. Rev. D 20, 2499 (1979).

9. W. G. Unruh, Phys. Rev. D 14, 870 (1976)

10. D. Bernard and A. Folacci, Phys. Rev. D 34, 2286 (1986) 